\title{
Left Anterior Apical Transition Zone of Prostate
}

National Cancer Institute

\section{Source}

National Cancer Institute. Left Anterior Apical Transition Zone of Prostate. NCI

Thesaurus. Code C128578.

The region of the prostate that is located on the anatomical left side of the anterior portion of the apical division of the transition zone. 